\title{
ON COLLECTIONWISE NORMALITY OF PRODUCT SPACES. II
}

\author{
KEIKO CHIBA
}

\begin{abstract}
In this paper the following results will be obtained. (1) Let $X$ be a closed image of a normal $M$-space and $Y$ a paracompact first countable $P$-space. If $X \times Y$ is normal, then $X \times Y$ is collectionwise normal. (2) Let $X$ be a collectionwise normal space and $Y$ a $\sigma$-locally compact paracompact space. If $X \times Y$ is normal, then $X \times Y$ is collectionwise normal.
\end{abstract}

1. Introduction. Throughout this paper all spaces are Hausdorff spaces and all maps are continuous.

In general, when $X$ and $Y$ are collectionwise normal spaces, $X \times Y$ is not necessarily normal. Further, the following fact is known.

(I) (PRZYMusińsKi [13]) (MA $+\neg \mathrm{CH})$. There exists a paracompact first countable space $X$ such that $X^{2}$ is normal but not collectionwise normal.

But, if we assume that $X \times Y$ is normal, then $X \times Y$ may be collectionwise normal. Concerning this, the following theorems are known.

(II) (RUDIN AND STARBIRD [15, 17]). Let $X$ be a collectionwise normal space and $Y$ a paracompact $M$-space and $X \times Y$ normal. Then $X \times Y$ is collectionwise normal.

(III) (NAGAMI [10]). Let $X$ be a collectionwise normal $P$-space and $Y$ a paracompact $\sigma$-space and $X \times Y$ normal. Then $X \times Y$ is collectionwise normal.

In this paper we shall prove the following results.

THEOREM 1. Let $X$ be a closed image of a normal $M$-space and $Y$ a paracompact first countable $P$-space and $X \times Y$ normal. Then $X \times Y$ is collectionwise normal.

THEOREM 2. Let $X$ be a collectionwise normal space and $Y$ a o-locally compact paracompact space and $X \times Y$ normal. Then $X \times Y$ is collectionwise normal.

The definitions of $M$-spaces and $P$-spaces are due to Morita [7] and $\sigma$-spaces are due to Okuyama [12].

Received by the editors June 20, 1983 and, in revised form, October 27, 1983. The author presented the contents of this paper at a summer seminar on July 27, 1982.

1980 Mathematics Subject Classification. Primary 54B10; Secondary 54D15.

Key words and phrases. Product space, normal, collectionwise normal, paracompact, first countable, $M$-space, $P$-space, $\sigma$-locally compact. 
2. Proof of Theorem 1. To prove Theorem 1, we use the following

(IV) [3]. Let $X$ be a collectionwise normal $\Sigma$-space (in the sense of Nagami [11]) and $Y$ a paracompact first countable $P$-space. Then $X \times Y$ is collectionwise normal.

We first prove the following lemmas. Lemma 1 is useful in proving collectionwise normality of a space $X$.

LEMma 1. Let $\left\{A_{\lambda} \mid \lambda \in \Lambda\right\}$ be a discrete family of closed subsets of a space $X$. If there exists a family $\left\{G_{\lambda, n} \mid \lambda \in \Lambda, n<\omega\right\}$ of open subsets of $X$ such that

(1) $\bigcup\left\{G_{\lambda, n} \mid n<\omega\right\} \supset A_{\lambda}$ for each $\lambda \in \Lambda$,

(2) $\operatorname{Cl}\left(G_{\lambda, n}\right) \cap A_{\mu}=\varnothing$ if $\lambda \neq \mu$,

(3) $\left\{G_{\lambda, n} \mid \lambda \in \Lambda\right\}$ is discrete in $X$,

then there exists a disjoint family $\left\{H_{\lambda} \mid \lambda \in \Lambda\right\}$ of open sets of $X$ such that $H_{\lambda} \supset A_{\lambda}$ for each $\lambda \in \Lambda$. Here $\omega$ denotes the first infinite ordinal.

Proof. Let us put $H_{\lambda}=\bigcup\left\{G_{\lambda, n}-\bigcup\left\{\mathrm{Cl}\left(G_{\mu, i}\right) \mid i \leqslant n, \mu \in \Lambda, \mu \neq \lambda\right\} \mid n<\omega\right\}$. Then $\left\{H_{\lambda} \mid \lambda \in \Lambda\right\}$ satisfies the required conditions.

LEMMA 2. Let $X$ and $Y$ be collectionwise normal spaces and $C$ a closed discrete subspace of $X$ and assume $X \times Y$ is normal. Let $\left\{A_{\lambda} \mid \lambda \in \Lambda\right\}$ be a discrete family of closed subsets of $X \times Y$. Then there exists a discrete family $\left\{G_{\lambda} \mid \lambda \in \Lambda\right\}$ of open sets of $X \times Y$ such that $G_{\lambda} \supset A_{\lambda} \cap(C \times Y)$ and $\mathrm{Cl}\left(G_{\lambda}\right) \cap A_{\mu}=\varnothing$ if $\lambda \neq \mu$.

Proof. ${ }^{1}$ Since $X \times Y$ is normal, there are open sets $H_{\lambda}$ in $X \times Y$ such that $A_{\lambda} \subset H_{\lambda}, \mathrm{Cl}\left(H_{\lambda}\right) \cap\left(\bigcup\left\{A_{\mu} \mid \mu \in \Lambda, \mu \neq \lambda\right\}\right)=\varnothing$. For each $x \in C$, there exists an open neighborhood $U(x)$ of $x$ such that $\{U(X) \mid x \in C\}$ is discrete in $X$. Let us put $A(\lambda, x)=\left\{y \in Y \mid(x, y) \in A_{\lambda}\right\}$ for each $x \in C$. Then $\{A(\lambda, x) \mid \lambda \in \Lambda\}$ is a discrete family of closed subsets of $Y$. Since $Y$ is collectionwise normal, there exist open sets $O(\lambda, x)$ in $Y$ such that $O(\lambda, x) \supset A(\lambda, x)$ for each $\lambda \in \Lambda$ and $\{O(\lambda, x) \mid \lambda \in \Lambda\}$ is discrete in $Y$. Then $G_{\lambda}=H_{\lambda} \cap\left(\cup_{x \in C} U_{x} \times O(\lambda, x)\right)$ has the desired properties.

Theorem 3 (Hoshina AND ChiBA). ${ }^{2}$ Let $X, Y$ and $Z$ be spaces such that $X \times Y$ is normal and $Y \times Z$ is collectionwise normal. Suppose $Y$ is first countable and there is $a$ closed onto map $f: Z \rightarrow X$ such that $X=\cup_{n<\omega} X_{n}, X_{n}$ is closed discrete for $n \geqslant 1$ and $f^{-1}(x)$ is countably compact for each $x \in X_{0}$. Then $X \times Y$ is collectionwise normal.

Proof. It is easy to see that $X$ and $Y$ are collectionwise normal. To prove collectionwise normality of $X \times Y$, let $\left\{A_{\lambda} \mid \lambda \in \Lambda\right\}$ be a discrete family of closed subsets of $X \times Y$. Then, by Lemma 2, for each $n \geqslant 1$, there exists a discrete family $\left\{G_{\lambda, n} \mid \lambda \in \Lambda\right\}$ of open sets of $X \times Y$ such that $G_{\lambda, n} \supset A_{\lambda} \cap\left(X_{n} \times Y\right)$ and $\mathrm{Cl}\left(G_{\lambda, n}\right)$ $\cap A_{\mu}=\varnothing$ if $\lambda \neq \mu$.

Let us put $B_{\lambda}=A_{\lambda}-\bigcup_{n=1}^{\omega} G_{\lambda, n}$. Then $B_{\lambda}$ is closed in $X \times Y$ and $B_{\lambda} \subset X_{0} \times Y$. Let us put $\phi=f \times 1_{Y}: Z \times Y \rightarrow X \times Y$ and $F_{\lambda}=\phi^{-1}\left(A_{\lambda}\right)$ for each $\lambda \in \Lambda$. Then $\left\{F_{\lambda} \mid \lambda \in \Lambda\right\}$ is a discrete family of closed subsets of $Z \times Y$. Since $Z \times Y$ is

\footnotetext{
${ }^{1}$ The referee has kindly pointed out this simple proof.

${ }^{2} \mathrm{~T}$. Hoshina and the author proved this theorem independently.
} 
collectionwise normal, there are open sets $H_{\lambda}$ in $Z \times Y$ such that $F_{\lambda} \subset H_{\lambda}$ and $H_{\lambda} \cap H_{\mu}=\varnothing$ if $\lambda \neq \mu$.

Let us put $A(\lambda, x)=\left\{y \in Y \mid(x, y) \in A_{\lambda}\right\}$ for each $x \in X_{0}$. For each $y \in$ $A(\lambda, x)$, there are an open set $W_{y}(x)$ in $Z$ and an open neighborhood $V_{x}(y)$ of $y$ in $Y$ such that $f^{-1}(x) \subset W_{y}(x)$ and $W_{y}(x) \times V_{x}(y) \subset H_{\lambda}$ because $f^{-1}(x)$ is countably compact and $Y$ is first countable. Since $f$ is a closed map, there is an open neighborhood $U_{y}(x)$ of $x$ in $X$ such that $f^{-1}\left(U_{y}(x)\right) \subset W_{y}(x)$.

Now let us put

$$
K_{\lambda, x}=\bigcup\left\{U_{y}(x) \times V_{x}(y) \mid y \in A(\lambda, x)\right\}
$$

and

$$
K_{\lambda}=\bigcup\left\{K_{\lambda, x} \mid x \in X_{0}\right\}
$$

Then we have:

(1) $K_{\lambda}$ is open in $X \times Y$.

(2) $\phi^{-1}\left(K_{\lambda}\right) \subset H_{\lambda}$.

(3) $A_{\lambda} \cap\left(X_{0} \times Y\right) \subset K_{\lambda}$.

Moreover, it is easy to see that:

(4) $K_{\lambda} \cap K_{\mu}=\varnothing$ if $\lambda \neq \mu$.

(5) $K_{\lambda} \cap A_{\mu}=\varnothing$ if $\lambda \neq \mu$.

Since $B_{\lambda} \subset K_{\lambda}$ and $\left\{B_{\lambda} \mid \lambda \in \Lambda\right\}$ is a discrete family of closed subsets of $X \times Y$, there is a discrete family $\left\{G_{\lambda, 0} \mid \lambda \in \Lambda\right\}$ of open sets of $X \times Y$ such that $B_{\lambda} \subset G_{\lambda, 0}$, $\mathrm{Cl}\left(G_{\lambda, 0}\right) \subset K_{\lambda}$ because $X \times Y$ is normal. Then we have $\mathrm{Cl}\left(G_{\lambda, 0}\right) \cap A_{\mu}=\varnothing$ if $\lambda \neq \mu$ by (5). Let us consider $\left\{G_{\lambda, n} \mid 0 \leqslant n<\omega\right\}$. Then, by Lemma 1 , there are open sets $P_{\lambda}$ in $X \times Y$ such that $A_{\lambda} \subset P_{\lambda}$ for each $\lambda \in \Lambda$ and $P_{\lambda} \cap P_{\mu}=\varnothing$ if $\lambda \neq \mu$. Hence the proof of collectionwise normality of $X \times Y$ is complete.

REMARK 1. In Theorem 3, if we replace the condition " $f^{-1}(x)$ is countably compact" by " $f^{-1}(x)$ is compact", then we can drop the first countability of $Y$.

Proof of Theorem 1. Let $Z$ be a normal $M$-space and $f: Z \rightarrow X$ a closed onto map and $Y$ a paracompact first countable $P$-space such that $X \times Y$ is normal. Then $Z \times Y$ is collectionwise normal by (IV) because every normal $M$-space is a collectionwise normal $\Sigma$-space [11, and in 12, Theorem 3.3]. By Theorem 3.1 in [5], $X=\cup_{n<\omega} X_{n}, X_{n}$ is closed discrete for $n \geqslant 1$ and $f^{-1}(x)$ is countably compact for each $x \in X_{0}$. Therefore, by Theorem 3, $X \times Y$ is collectionwise normal.

THEOREM 4. Let $X$ be a collectionwise normal $\Sigma$-space and $Y$ a closed image of a paracompact first countable $M$-space and $X \times Y$ is normal. Then $X \times Y$ is collectionwise normal.

Proof. Let $X$ be a collectionwise normal $\Sigma$-space and $Z$ a paracompact first countable $M$-space and $f: Z \rightarrow Y$ a closed onto map such that $X \times Y$ is normal. Then $X \times Z$ is collectionwise normal by (IV) because every $M$-space is a $P$-space [7]. By [4], $Y=\cup_{n<\omega} Y_{n}, Y_{n}$ is closed discrete for $n \geqslant 1$ and $f^{-1}(y)$ is compact for each $y \in Y_{0}$. Hence $X \times Y$ is collectionwise normal (see Remark 1).

We proved essentially the following in [2, Theorem 6]. 
(V). Let $X$ be a locally countably compact space and $Z$ a first countable space and $f: Z \rightarrow Y$ a closed map onto a space $Y$ such that $Y=\cup_{n<\omega} Y_{n}, Y_{n}$ is closed discrete for $n \geqslant 1$ and $f^{-1}(y)$ is compact for each $y \in Y_{0}$. If $X \times Z$ is normal, then $X \times Y$ is normal.

The following theorem can be proved in a way similar to the proof of Theorem 4 using (V).

THEOREM 5. Let $X$ be a locally countably compact collectionwise normal $\Sigma$-space and $Y$ a closed image of a paracompact first countable $M$-space. Then $X \times Y$ is collectionwise normal.

REMARK 2. Let $X$ and $Y$ be spaces in Theorem 1 or 4 . It is not known whether $X \times Y$ is normal or not.

3. Proof of Theorem 2 and a related result. Even in case $Y$ has only one nonisolated point, $X \times Y$ is not necessarily normal for a collectionwise normal space $X$. In fact, let $X$ be the Dowker space constructed by Rudin [14] and $Y=[0, \omega]$ with the order topology. Then $X$ is collectionwise normal and $X \times Y$ is not normal [14]. However, in case $Y$ has only one nonisolated point, the following holds. "For any collectionwise normal space $X$, if $X \times Y$ is normal, then $X \times Y$ is collectionwise normal."

We shall prove the following theorem which contains both in case (i) $Y$ is $\sigma$-locally compact paracompact, and in case (ii) $Y$ has only one nonisolated point.

THEOREM 6. Let $X$ be a collectionwise normal space and $Y$ a paracompact space such that $Y=\left(\cup_{n<\omega} C_{n}\right) \cup D$ where $C_{n}$ is a locally compact closed subspace of $Y$ for each $n<\omega$ and for each $y \in D$ is an isolated point of $Y$. If $X \times Y$ is normal, then $X \times Y$ is collectionwise normal.

There exist spaces expressed as the form of $Y$ in Theorem 6 but neither (i) nor (ii). E. g., the Michael line [6] is so. Further, if $Y$ is a paracompact subspace of $F$ where $F$ is the Bing's Example $\mathrm{G}$ or $\mathrm{H}$ [1], then $Y$ satisfies the conditions in Theorem 6 but $Y$ is necessarily neither (i) nor (ii). Therefore it seems good to consider the space $Y$ satisfying the conditions in Theorem 6.

A subspace $A$ of a space $X$ is said to be $P$-embedded in $X$ if every locally finite cozero-set cover of $A$ has a refinement which can be extended to a locally finite cozero-set cover of $X$ (Shapiro [16]).

LEMMA 3. Let $X$ and $Y$ be collectionwise normal spaces and $Y$ is expressed as $Y=C \cup D$ such that $C \cap D=\varnothing$ and each $y \in D$ is an isolated point of $Y$. Let $X \times Y$ be normal and $\left\{A_{\lambda} \mid \lambda \in \Lambda\right\}$ a discrete family of closed subsets of $X \times Y$. If there exists a disjoint family $\left\{G_{\lambda} \mid \lambda \in \Lambda\right.$ \} of open sets of $X \times Y$ such that $G_{\lambda} \supset A_{\lambda} \cap$ $(X \times C)$, then there exists a disjoint family $\left\{M_{\lambda} \mid \lambda \in \Lambda\right\}$ of open sets of $X \times Y$ such that $M_{\lambda} \supset A_{\lambda}$.

Proof. We can choose a discrete family $\left\{E_{\lambda} \mid \lambda \in \Lambda\right\}$ of open sets of $X \times Y$ such that $A_{\lambda} \cap(X \times C) \subset E_{\lambda}, \mathrm{Cl}\left(E_{\lambda}\right) \subset G_{\lambda}-\bigcup_{\mu \neq \lambda} A_{\mu}$. Let us put $D_{\lambda}=A_{\lambda}-E_{\lambda}$. Then $\left\{D_{\lambda} \mid \lambda \in \Lambda\right\}$ is a discrete family of closed subsets of $X \times D$. Since $X \times D$ is collectionwise normal, there is a disjoint family $\left\{J_{\lambda} \mid \lambda \in \Lambda\right\}$ of open sets of $X \times D$ 
such that $D_{\lambda} \subset J_{\lambda}$. We remark that $J_{\lambda}$ is open in $X \times Y$. Since $D_{\lambda}$ and $\bigcup_{\mu \neq \lambda} \operatorname{Cl}\left(E_{\mu}\right)$ are disjoint closed subsets of $X \times Y$ and $X \times Y$ is normal, there are open sets $K_{\lambda}$ and $L_{\lambda}$ in $X \times Y$ such that $D_{\lambda} \subset K_{\lambda}, \cup_{\mu \neq \lambda} \mathrm{Cl}\left(E_{\mu}\right) \subset L_{\lambda}$ and $K_{\lambda} \cap L_{\lambda}=\varnothing$. Let us put $M_{\lambda}=E_{\lambda} \cup\left(J_{\lambda} \cap K_{\lambda}\right)$. Then $M_{\lambda}$ is open in $X \times Y$. Moreover, it is easy to see that $M_{\lambda} \supset A_{\lambda}$ and $\left\{M_{\lambda} \mid \lambda \in \Lambda\right\}$ is disjoint.

Proof of Theorem $6 .^{3}$ Let $X$ and $Y$ be spaces in Theorem 6. Let $\left\{A_{\lambda} \mid \lambda \in \Lambda\right\}$ be a discrete family of closed subsets of $X \times Y$. For $n<\omega, X \times C_{n}$ is collectionwise normal by (II) in $\S 1$. Therefore, for each $n<\omega$, there is a discrete family $\left\{J_{\lambda, n} \mid \lambda \in\right.$ $\Lambda$ \} of open sets of $X \times C_{n}$ such that $A_{\lambda} \cap\left(X \times C_{n}\right) \subset J_{\lambda, n}$ for each $\lambda \in \Lambda$.

Also $X \times C_{n}$ is $P$-embedded in $X \times Y$ by Theorem 5.2 in [16] and Theorem 4 in [8]. Thus, by Theorem 2.4 in [9], there is a locally finite family $\left\{H_{\lambda, n}^{\prime} \mid \lambda \in \Lambda\right\}$ of cozero-sets of $X \times Y$ such that

$$
A_{\lambda} \cap\left(X \times C_{n}\right) \subset H_{\lambda, n}^{\prime} \cap\left(X \times C_{n}\right) \subset J_{\lambda, n} \quad \text { for each } \lambda \in \Lambda \text {. }
$$

Let $H_{\lambda, n}$ be open sets of $X \times Y$ such that $A_{\lambda} \cap\left(X \times C_{n}\right) \subset H_{\lambda, n}, \mathrm{Cl}\left(H_{\lambda, n}\right) \subset H_{\lambda, n}^{\prime}$. Let us put $L_{\lambda, n}=H_{\lambda, n}-\bigcup_{\mu \neq \lambda} \mathrm{Cl}\left(H_{\mu, n}\right)$. Then $L_{\lambda, n}$ is open in $X \times Y, A_{\lambda} \cap(X \times$ $\left.C_{n}\right) \subset L_{\lambda, n}$ for each $\lambda \in \Lambda$ and $\left\{L_{\lambda, n} \mid \lambda \in \Lambda\right\}$ is disjoint.

Since $X \times Y$ is normal, there is a discrete family $\left\{G_{\lambda, n} \mid \lambda \in \Lambda\right\}$ of open sets of $X \times Y$ such that $A_{\lambda} \cap\left(X \times C_{n}\right) \subset G_{\lambda, n}, \operatorname{Cl}\left(G_{\lambda, n}\right) \subset L_{\lambda, n}-\cup_{\mu \neq \lambda} A_{\mu}$. If we consider $\left\{G_{\lambda, n} \mid \lambda \in \Lambda, n<\omega\right\}$, by Lemma 1 , there is a disjoint family $\left\{G_{\lambda} \mid \lambda \in \Lambda\right\}$ of open sets of $X \times Y$ such that $G_{\lambda} \supset A_{\lambda} \cap\left[X \times\left(\cup_{n<\omega} C_{n}\right)\right]$. By Lemma 3, there exists a disjoint family $\left\{M_{\lambda} \mid \lambda \in \Lambda\right\}$ of open sets of $X \times Y$ such that $M_{\lambda} \supset A_{\lambda}$. Therefore $X \times Y$ is collectionwise normal. The proof of Theorem 6 is complete.

\section{REFERENCES}

1. R. H. Bing, Metrization of topological spaces, Canad. J. Math. 3 (1951), 175-186.

2. K. Chiba, On products of normal spaces, Rep. Fac. Sci. Shizuoka Univ. 9 (1974), 1-11.

3. __ On collectionwise normality of product spaces. I, Proc. Amer. Math. Soc.91 (1984), 649-652.

4. V. V. Fillippov, On feathered paracompacts, Soviet Math. Dokl. 9 (1968), 161-164.

5. T. Ishii, wM-spaces and closed maps, Proc. Japan Acad. 46 (1970), 16-21.

6. E. Michael, The product of a normal space and a metric space need not be normal, Bull. Amer. Math. Soc. 69 (1963), 375-376.

7. K. Morita, Products of normal spaces with metric spaces, Math. Ann. 154 (1964), 365-382.

8. On the dimension of the product of topological spaces, Tsukuba J. Math. 1 (1977), 1-6.

9. K. Morita and T. Hoshina, P-embedding and product spaces, Fund. Math. 93 (1976), 71-80.

10. K. Nagami, $\sigma$-spaces and product spaces, Math. Ann. 181 (1969), 109-118.

11. ¿-spaces, Fund. Math. 65 (1969), 169-192.

12. A. Okuyama, Some generalizations of metric spaces, their metrization theorems and product spaces, Sci. Rep. Tokyo Kyoiku Daigaku 9 (1967), 236-254.

13. T. Przymusiński, A Lindelöf space $X$ such that $X^{2}$ is normal but not paracompact, Fund. Math. 78 (1973), 291-296.

14. M. E. Rudin, The normal space $X$ for which $X \times I$ is not normal, Fund. Math. 73 (1971), 179-186.

15. M. E. Rudin and M. Starbird, Products with a metric factor, Gen. Topology Appl. 5 (1975), 235-248.

16. H. L. Shapiro, Extensions of pseudometrics, Canad. J. Math. 18 (1966), 981-998.

17. M. Starbird, The normality of products with a compact or a metric factor, $\mathrm{Ph}$. D. Thesis, University of Wisconsin, 1974.

\footnotetext{
Department of Mathematics, Faculty of Science, Shizuoka University, Ohya, Shizuoka 422, JAPAN
}

\footnotetext{
${ }^{3}$ The author first proved Theorem 6 by another method. T. Hoshina pointd out the proof can be made simpler by using the idea of $P$-embedding.
} 